hep-th/0504126

\title{
5D Black Rings and 4D Black Holes
}

\author{
Davide Gaiotto* , Andrew Strominger* and Xi Yin* \\ Center of Mathematical Sciences \\ Zhejiang University, Hangzhou 310027 China
}

\begin{abstract}
It has recently been shown that the M theory lift of a IIA 4D BPS Calabi-Yau black hole is a 5D BPS black hole spinning at the center of a Taub-NUT-flux geometries, and a certain linear relation between 4D and 5D BPS partition functions was accordingly proposed. In the present work we fortify and enrich this proposal by showing that the M-theory lift of the general 4D multi-black hole geometry are 5D black rings in a TaubNUT-flux geometry.
\end{abstract}

\footnotetext{
* Permanent address: Jefferson Physical Laboratory, Harvard University, Cambridge, MA,
} USA. 


\section{Contents}

1. Introduction . . . . . . . . . . . . . . . . . . . . . . . . . . . . . . . . . 1

2. Multicenter BPS solutions in 4D . . . . . . . . . . . . . . . . . . . . . . 2

3. $5 \mathrm{D}$ solutions ............................. 4

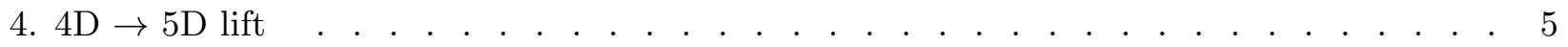

\section{Introduction}

In a recent paper [1] it was shown that the M-theory lift of a general 4D BPS CalabiYau black hole can be viewed as a 5D BPS spinning black hole [2] sitting in the center of a flux-Taub-NUT spacetime. This suggested a direct relation between the microstates of $4 \mathrm{D}$ and 5D black holes, and (invoking [3]) motivated a conjecture of the form

$$
Z_{5 D}=Z_{4 D}=\left|Z_{t o p}\right|^{2}
$$

relating a 4D BPS partition function, a 5D BPS partition function and the topological string. See [1] for the precise form of and arguments appearing in (1.1).

A recent surprise [4] is that BPS objects in 5D include black rings as well as black holes. The $5 \mathrm{D}$ partition function $Z_{5 D}$ should include all BPS states, in particular black rings. A check of our conjecture is then that $Z_{4 D}$ must include a contribution to match the $5 \mathrm{D}$ black rings in $Z_{5 D}$.

Indeed we will show that there is just such a matching contribution. In a series of beautiful papers [5, 66,7] Denef and collaborators have explicitly constructed multi-center 4D BPS black hole solutions which in general carry angular momenta. The black holes in these solutions can have different sets of charges and they are bound to one another in the sense that the black holes separations are fixed in terms of their charges and the asymptotic values of the moduli. In this paper we will construct exact "flux-Taub-NUT-black-ring" solutions describing a black ring in Taub-NUT with four-form flux turned on. We further show that these solutions are precisely the lift to 5D of the Denef multi-center solutions, and the $4 \mathrm{D}$ black hole separations become the radii of the $5 \mathrm{D}$ rings. This result fortifies and enriches the 4D-5D connection proposed in [1], and will also clearly have implications for our understanding of the topological string 1

1 In particular we expect the anomalous background dependence of the topological string to be equivalent to the anomalous asymptotic moduli dependence of the black hole partition function arising from split attractor flows as analyzed in [8,9, 10,7, 7, 5, 6]. 
This paper is organized as follows. Section 2 reviews the multi-center black hole solutions. Section 3 constructs a general solution of 5D supergravity describing black rings and black holes in a multi-Taub-NUT-flux geometry. In section 4 we show that section 3 is the M-theory lift of section 2, and describe the basic example of lifting the bound state of a D6-brane with a D4-D2-D0 black hole to a 5D black ring.

\section{Multicenter BPS solutions in 4D}

In this section we review, and slightly reformulate, the general multi-center BPS solution of $4 \mathrm{D} \mathcal{N}=2$ supergravity? found in [7]. The solution is characterized by electromagnetic charges and asymptotic moduli. It may be expressed in terms of $2 h_{11}+2$ real harmonic functions on $R^{3}$

$$
\left(\begin{array}{c}
H^{\Lambda}(\vec{x}) \\
H_{\Lambda}(\vec{x})
\end{array}\right)=\left(\begin{array}{c}
h^{\Lambda} \\
h_{\Lambda}
\end{array}\right)+\sum_{s}\left(\begin{array}{c}
p_{i}^{\Lambda} \\
q_{\Lambda, i}
\end{array}\right) \frac{1}{\left|\vec{x}-\vec{x}_{i}\right|}
$$

where $\left(p_{i}^{\Lambda}, q_{\Lambda i}\right)$ is the electromagnetic charge located at the spatial position $\vec{x}_{i}$, and $\left(h^{\Lambda}, h_{\Lambda}\right)$ are constants which will shortly be related to the asymptotic moduli. The projective scalar moduli $X^{\Lambda}$ as a function of spatial positions are then given by

$$
C X^{\Lambda}(\vec{x})=H^{\Lambda}(\vec{x})+\left.\frac{i}{\pi} \frac{\partial S_{b h}\left(p^{\Lambda}, q_{\Lambda}\right)}{\partial q_{\Lambda}}\right|_{p^{\Lambda}=H^{\Lambda}(\vec{x}), q_{\Lambda}=H_{\Lambda}(\vec{x})} .
$$

The complex function $C$ depends on the choice of projective gauge (and may be set to one by an appropriate choice). As a function of the moduli and prepotential (or periods $F_{\Lambda}$ ) $S_{b h}$ here is given by

$$
S_{b h}\left(C X^{\Lambda}(\vec{x})\right)=\frac{\pi}{2} \operatorname{Im}\left[C X^{\Lambda} \bar{C}_{\bar{F}}\right] .
$$

In order to find $S_{b h}$ as a function of charges, as needed in (2.2), one must solve the algebraic attractor equations [11,12. This may or may not be analytically possible, depending on the form of the prepotential and the charge vector. Note that the $S_{b h}$ used here is a function of position and is equal to the black hole entropy only at the horizon. The constants $h$ encode the values of the moduli at spatial infinity, i.e. $\operatorname{Re}\left[C X^{\Lambda}(\infty)\right]=h^{\Lambda}, \operatorname{Re}\left[C F^{\Lambda}(\infty)\right]=h_{\Lambda}$.

2 The solutions reviewed here solve the leading order equations, and do not incorporate $R^{2}$ corrections. 
Given the moduli fields $X^{\Lambda}(\vec{x})$ the four dimensional metric is then simply

$$
d s_{4}^{2}=-\frac{\pi}{S_{b h}}\left(d t+\omega^{(4)}\right)^{2}+\frac{S_{b h}}{\pi} d \vec{x}^{2}
$$

where it is implicit that $S_{b h}=S_{b h}\left(X^{\Lambda}(\vec{x})\right)$ and $\omega^{(4)}$ is the solution of

$$
d \omega^{(4)}=H_{\Lambda} *^{3} d H^{\Lambda}-H^{\Lambda} *^{3} d H_{\Lambda} .
$$

The gauge fields strengths are

$$
d A^{\Lambda}=d\left[S_{b h}^{-1} \frac{\partial S_{b h}}{\partial q_{\Lambda}}\left(d t+\omega^{(4)}\right)\right]_{p^{\Lambda}=H^{\Lambda}, q_{\Lambda}=H_{\Lambda}}+*^{3} d H^{\Lambda}
$$

Finally the equilibrium positions $\vec{x}_{j}$ of the black hole centers are determined by the asymptotic moduli and the charges via the integrability condition following from (2.5), which may be written

$$
\left.\left[p_{j}^{\Lambda} H_{\Lambda}(\vec{x})-q_{j \Lambda} H^{\Lambda}(\vec{x})\right]\right|_{\vec{x}=\vec{x}_{j}}=0 .
$$

The basic example of this paper, which illustrates the connection to the black ring, is a bound state of a single $D 6$ brane at $r=0$ and a $D 4-D 2-D 0$ black hole with charges $\left(p^{A}, q_{A}, q_{0}\right)$ at $r=L, \theta=0$. The harmonic functions are:

$$
\begin{aligned}
& H^{0}=\frac{4}{R_{T N}^{2}}+\frac{1}{r} \\
& H^{A}=\frac{p^{A}}{\left(r^{2}+L^{2}-2 r L \cos \theta\right)^{\frac{1}{2}}} \\
& H_{A}=h_{A}+\frac{q_{A}}{\left(r^{2}+L^{2}-2 r L \cos \theta\right)^{\frac{1}{2}}} \\
& H_{0}=-\frac{q_{0}}{L}+\frac{q_{0}}{\left(r^{2}+L^{2}-2 r L \cos \theta\right)^{\frac{1}{2}}}
\end{aligned}
$$

The integrability condition $(2.7)$ is then

$$
\frac{1}{L}+\frac{4}{R_{T N}^{2}}=\frac{h_{A} p^{A}}{q_{0}}
$$

As the parameter $R_{T N}$ goes to infinity the distance between the centers reaches a minimum value, while for $R_{T N}$ small enough the distance between the centers will diverge, and the bound state disappears. 


\section{5D solutions}

This section will describe some new supersymmetric 5D black ring-Taub-NUT-flux solutions which generalize previous solutions of minimal supergravity [4, 13]. In the next section we will see they are simply the lift to 5D of the 4D multicenter solutions reviewed in the previous section.

$\mathcal{N}=2$ supergravity fields in $5 \mathrm{D}$ are organized by the so-called very special geometry [14], parameterized by $h_{11}$ real scalar fields $Y^{A}$, subject to the constraint

$$
D_{A B C} Y^{A} Y^{B} Y^{C}=1
$$

for constant couplings $D_{A B C}$. It is useful to further define

$$
Y_{A} \equiv 3 D_{A B C} Y^{B} Y^{C}
$$

BPS solutions in 5D $\mathcal{N}=2$ supergravity may be written following [15, 16, 17, 13

$$
\begin{aligned}
& d s_{5}^{2}=-2^{-4 / 3} f^{-2}(d t+\omega)^{2}+2^{2 / 3} f d s_{X}^{2} \\
& F^{A}=d\left[f^{-1} Y^{A}(d t+\omega)\right]+\Theta^{A}
\end{aligned}
$$

where $d s_{X}^{2}$ is hyperkäler metric on a $4 \mathrm{D}$ hyperkäler space $X, \Theta^{A}$ are closed self-dual 2-forms on $X$, the self-dual part of $d \omega$ is $-f Y_{A} \Theta^{A}$ and $f$ is a function on $X$ obeying

$$
\nabla^{2}\left(f Y_{A}\right)=3 D_{A B C} \Theta^{B} \cdot \Theta^{C}
$$

When the space $X$ is Taub-NUT, 3 one has

$$
d s_{X}^{2}=H^{0}(\vec{x}) d \vec{x}^{2}+H^{0}(\vec{x})^{-1}\left(d x^{5}+\omega^{0}\right)^{2}, \quad d \omega^{0}=*^{3} d H^{0},
$$

with $H^{0}$ a harmonic function as in (2.8), and the coordinate $x^{5}$ has periodicity $4 \pi$. Closed self-dual 2-forms are then given by

$$
\Theta^{A}=d\left[\frac{H^{A}}{H^{0}}\left(d x^{5}+\omega^{0}\right)\right]+*^{3} d H^{A},
$$

with harmonic $H^{A}$ as in (2.8). Inserting (3.6) the equation (3.4) for $f$ becomes

$$
\nabla^{2}\left(f Y_{A}\right)=6 D_{A B C} \nabla\left(\frac{H^{B}}{H^{0}}\right) \cdot \nabla\left(\frac{H^{C}}{H^{0}}\right)
$$

3 The more general solution with $X$ being a Gibbons-Hawking space was presented in [17. 
This is magically solved by

$$
f Y_{A}=H_{A}+\frac{3 D_{A B C} H^{B} H^{C}}{H^{0}} .
$$

$f$ and $Y_{A}$ are then determined by (3.1) and (3.8). This immediately gives the self-dual part of $d \omega$. It is straightforward to show that $\omega$ can be written

$$
\omega=-\left(H_{0}+2 \frac{D_{A B C} H^{A} H^{B} H^{C}}{\left(H^{0}\right)^{2}}+\frac{H_{A} H^{A}}{H^{0}}\right)\left(d x^{5}+\omega^{0}\right)+\omega^{(4)},
$$

where $\omega^{(4)}$ satisfies the integrability condition (2.7).

As we will see more clearly in the next section, the solution (3.3) together with (3.6), (3.8),(3.9) describe black rings in the Taub-NUT space.

\section{4. $4 \mathrm{D} \rightarrow 5 \mathrm{D}$ lift}

$\mathcal{N}=25 \mathrm{D}$ supergravity may be viewed as the circle decompactification of $\mathcal{N}=24 \mathrm{D}$ supergravity. In this section we see that the 5D black ring solution of the previous section is the lift from $4 \mathrm{D}$ of the general multi-center black hole solutions. A discussion of the relevant geometry is in [18].

Given a solution of $d s_{4 D}^{2}, A_{4 D}^{A}, A_{4 D}^{0}, z^{A}=\frac{X^{A}}{X^{0}}$ of $4 \mathrm{D}$ supergravity, a solution of $5 \mathrm{D}$ supergravity is quite generally given by

$$
\begin{aligned}
d s_{5 D}^{2} & =2^{2 / 3} \mathcal{V}^{2}\left(d x^{5}+A_{4 D}^{0}\right)^{2}+2^{-1 / 3} \mathcal{V}^{-1} d s_{4 D}^{2}, \\
A_{5 D}^{A} & =A_{4 D}^{A}+\operatorname{Re} z^{A}\left(d x^{5}+A_{4 D}^{0}\right), \\
Y^{A} & =\mathcal{V}^{-1} \operatorname{Im} z^{A}, \quad \mathcal{V} \equiv\left(D_{A B C} \operatorname{Im} z^{A} \operatorname{Im} z^{B} \operatorname{Im} z^{C}\right)^{\frac{1}{3}} .
\end{aligned}
$$

Inserting the multi-center $4 \mathrm{D}$ solution of section 2 then gives the general black ring solutions of section 3 .

To be more explicit, let us consider the $4 \mathrm{D}$ prepotential

$$
F(X)=\frac{D_{A B C} X^{A} X^{B} X^{C}}{X^{0}}
$$

The expression for the entropy as a function of the charges is known, although complicated [19]. It is

$$
\begin{aligned}
& S(p, q)=2 \pi \sqrt{Q^{3} p^{0}-J^{2}\left(p^{0}\right)^{2}}, \\
& Q^{\frac{3}{2}}=D_{A B C} y^{A} y^{B} y^{C}, \\
& 3 D_{A B C} y^{A} y^{B}=q_{C}+\frac{3 D_{A B C} p^{A} p^{B}}{p^{0}}, \\
& J=\frac{q_{0}}{2}+\frac{D_{A B C} p^{A} p^{B} p^{C}}{\left(p^{0}\right)^{2}}+\frac{p^{A} q_{A}}{2 p^{0}} .
\end{aligned}
$$


Correspondingly there will be certain functions $Q(\vec{x})$ and $J(\vec{x})$ built out of the harmonic functions $\left(H^{\Lambda}(\vec{x}), H_{\Lambda}(\vec{x})\right)$. The volume of the Calabi-Yau at $\vec{x}$ is $\mathcal{V}(\vec{x})^{3}$, with

$$
\mathcal{V}(\vec{x})=\frac{S\left(H^{\Lambda}, H_{\Lambda}\right)}{2 \pi H^{0} Q}
$$

With a bit algebra, (4.1) then yields for the metric

$$
\begin{aligned}
& d s_{5 D}^{2}=-2^{-4 / 3} Q(\vec{x})^{-2}\left(d t+\omega^{(4)}-2 J(\vec{x})\left(d x^{5}+\omega^{0}\right)\right)^{2}+2^{2 / 3} Q(\vec{x}) d s_{T N}^{2} \\
& d s_{T N}^{2}=H^{0}(\vec{x}) d \vec{x}^{2}+H^{0}(\vec{x})^{-1}\left(d x^{5}+\omega^{0}\right)^{2}
\end{aligned}
$$

where

$$
d \omega^{0}=*^{3} d H^{0}
$$

and $\omega^{(4)}$ is given by (2.5). This is precisely the solution we found in section 3 . The $4 \mathrm{D}$ multi-centered black hole solution in general has several horizons, which lifts to horizons in 5D. These horizons are circle bundles over $S^{2}$. Depending on whether each center has nonzero magnetic KK charge, the 5D horizons will be either quotients of $S^{3}$, corresponding to a $5 \mathrm{D}$ spinning black hole, or $S^{2} \times S^{1}$, corresponding to a black ring.

Now let us further specialize to the bound state of a single D6-brane with a D4-D2-D0 black hole with charges $\left(p^{A}, q_{A}, q_{0}\right)$. The relevant harmonic functions are given in (2.8). In the limit $R_{T N} \rightarrow \infty, H^{0} \rightarrow \frac{1}{r}$, (4.5) becomes precisely the black ring solution in flat $5 \mathrm{D}$ spacetime [4]. The radius of the ring is $R_{\text {ring }}=L$. It is constructed from wrapped M5 branes with charges $p^{A}$, carries M2 charges $\tilde{q}_{A}=q_{A}+3 D_{A B C} p^{B} p^{C}$ and $S U(2)_{L}$ spin $J_{L}=q_{0} / 2$.

Note that the entropy of the two-centered black hole comes from only the D4-D2-D0 system. It is amusing to verify directly that the tree level entropy of the D4-D2-D0 system

of charge $\left(p^{A}, q_{A}, q_{0}\right)$ indeed agrees with that of the black ring [20], 21] with M5-M2 charge $\left(p^{A}, \tilde{q}_{A}\right)$ and angular momentum $J_{L}=q_{0} / 2$.

More generally, when there are mulitple 4D black holes carrying D6 charge, the background geometry of the 5D lift will be a resolved multi-Taub-Nut-flux geometry. The black holes of the 4D solution that carry $D 6$ charges will lift to 5D spinning black holes at the fixed points of the $U(1)_{L}$ isometry of the multi-Taub-NUT background. Those that do not will lift to $5 \mathrm{D}$ black rings tracing orbits of the isometry.

\section{Acknowledgements}

We would like to thank M. Guica for useful conversations. This work was supported in part by DOE grant DE-FG02-91ER40654. 


\section{References}

[1] D. Gaiotto, A. Strominger and X. Yin, "New Connections Between 4D and 5D Black Holes," arXiv:hep-th/0503217.

[2] J.C. Breckenridge, R.C. Myers, A.W. Peet and C. Vafa, "D-branes and Spinning Black Holes", hep-th/9602065.

[3] H. Ooguri, A. Strominger and C. Vafa, "Black hole attractors and the topological string," arXiv:hep-th/0405146.

[4] H. Elvang, R. Emparan, D. Mateos and H. S. Reall, "A supersymmetric black ring," Phys. Rev. Lett. 93, 211302 (2004) arXiv:hep-th/0407065.

[5] F. Denef, "Supergravity flows and D-brane stability," JHEP 0008, 050 (2000) arXiv:hep-th/0005049.

[6] F. Denef, B. R. Greene and M. Raugas, "Split attractor flows and the spectrum of BPS D-branes on the quintic," JHEP 0105, 012 (2001) arXiv:hep-th/0101135.

[7] B. Bates and F. Denef, "Exact solutions for supersymmetric stationary black hole composites," arXiv:hep-th/0304094.

[8] G. W. Moore, "Attractors and arithmetic," arXiv:hep-th/9807056.

[9] G. W. Moore, "Arithmetic and attractors," arXiv:hep-th/9807087.

[10] G. W. Moore, "Les Houches lectures on strings and arithmetic," arXiv:hep-th/0401049.

[11] S. Ferrara, R. Kallosh and A. Strominger, "N=2 extremal black holes," Phys. Rev. D 52, 5412 (1995) arXiv:hep-th/9508072.

[12] A. Strominger, "Macroscopic Entropy of $N=2$ Extremal Black Holes," Phys. Lett. B 383, 39 (1996) arXiv:hep-th/9602111.

[13] P. Kraus and F. Larsen, "Attractors and black rings," arXiv:hep-th/0503219.

[14] M. Gunaydin, G. Sierra and P. K. Townsend, "Gauging The D = 5 Maxwell-Einstein Supergravity Theories: More On Jordan Algebras," Nucl. Phys. B 253, 573 (1985).

[15] J. P. Gauntlett, J. B. Gutowski, C. M. Hull, S. Pakis and H. S. Reall, "All supersymmetric solutions of minimal supergravity in five dimensions," Class. Quant. Grav. 20, 4587 (2003) arXiv:hep-th/0209114.

[16] J. B. Gutowski and H. S. Reall, "General supersymmetric AdS(5) black holes," JHEP 0404, 048 (2004) arXiv:hep-th/0401129.

[17] J. P. Gauntlett and J. B. Gutowski, "General concentric black rings," Phys. Rev. D 71, 045002 (2005) arXiv:hep-th/0408122.

[18] R. Gregory, J. A. Harvey and G. W. Moore, "Unwinding strings and T-duality of Kaluza-Klein and H-monopoles," Adv. Theor. Math. Phys. 1, 283 (1997) arXiv:hepth/9708086].

[19] M. Shmakova, 'Calabi-Yau black holes," Phys. Rev. D 56, 540 (1997) arXiv:hepth/9612076]. 
[20] I. Bena and P. Kraus, "Microscopic description of black rings in AdS/CFT," JHEP 0412, 070 (2004) arXiv:hep-th/0408186.

[21] M. Cyrier, M. Guica, D. Mateos and A. Strominger, "Microscopic entropy of the black ring," arXiv:hep-th/0411187. 